\title{
Immobility Edge-Method of Plain Graph
}

\author{
Liping Zhang \& Qiu Yu \\ Shanghai University of Engineering Science, Shanghai 201620, China \\ E-mail: yuqiu@vip.citiz.net
}

Received: November 9, 2011 Accepted: November 28, 2011 Published: February 1, 2012

doi:10.5539/jmr.v4n1p49 URL: http://dx.doi.org/10.5539/jmr.v4n1p49

\begin{abstract}
This article introduced the immobility edge-method for the calculation and analysis of color function. Meanwhile it give out an example to calculate the color function of a graph with 4-cycle and explained the reducibility for the configuration of it, which is a different method compare with others.
\end{abstract}

Keywords: Discompose methods of similarities and differences, Color number reverse, Immobility edges

\section{Introduction}

The prove of the four color theorem usually introduces a plane triangulations graph $T$, any plane graph which has the vertexes less than $T$ graph is a 4-chromatic graph, while graph $T$ is not.Meanwhile give out a group graphs of unavoidable complete set as shown in figure 1 below.

$<$ Figure $1>$

\subsection{Discompose methods of similarity and difference}

As shown in figure 2 below, for the 4-cycle which one edge is moved, the colors of the two corresponding vertices (indicated by air core circle) have two possible cases: similarity and difference for their colors. If the colors of the two vertices are difference, it is equal to a 4-cycle; if the color of the two vertexes is the same, it is equal to a 3-cycle. So these two cases which are added together equals to the former one on the left in figure 2 (a), move the picture will have another equation in figure 2 (b). In this article this method is regarded as the "discompose methods of similarity and difference".

$<$ Figure $2>$

From the figure 2(b) above, we know that this method is true for any kinds of plain graph. And the discompose result $P$ on right side of the equality sign in figure 2(b) is the one with the color number bigger than or equal to zero. For the color number of $P_{1}$ is always bigger than or equal to that of $P_{2}$. Let us suppose that $P(\mathrm{G}+\mathrm{e})$ stands for the graph $\mathrm{G}$ with one edge more and also its color function and color number, and $P(\mathrm{G})$ stands for the graph $\mathrm{G}$ with one edge moved. $P(\mathrm{G}-\mathrm{v})$ stand for the graph with one edge moved and the edge of its two corresponding vertices merged. Then we have the color function below:

$$
P(G+e)=P(G)-P(G-v) \geq 0
$$

Hence we know these equations below will never happen:

$$
P(G+e)=P(G)-P(G-v)<0, \quad P(G)<P(G-v), \quad P(G)<P(G+e)
$$

These equations above do not exist for plain graph and even a non-plain one. According to the physical concept this phenomenon is not true. So it is regarded as "color number reverse".

\subsection{The color functions of graph with tree structure alike}

As shown in figure 3 (a) below, it is a plain figure with a structure of a tree. In figure 3 (b), it has only one 3-cycle, except this, it is a structure of a tree. The color functions for them are written below:

$$
P_{a}=36 \times 3^{m-3}, \quad P_{b}=24 \times 3^{m-3}
$$

Here $m$ stands for the number of the vertexes. We regard these structures in the figure 3 as the tree like structure.

$<$ Figure $3>$ 


\section{Discompose Methods of Immovable Vertices}

\subsection{Discompose example of 4-cycle and 5-cycle}

Just make a rule that two edges (thick line in figure 4) do not take part in the discompose action. These two edges are considered as immovable edges. Other vertices linked with edges will be discomposed continuously until it forms two kinds of graphs with tree like structure as shown in figure 3. Below in figure 4(a) and (b) are examples graph of 4-cycle and 5-cycle for the discompose action. One kind of graphs is the structure of some trees, then adding all the color functions of these trees to get one with a coefficient to stand for and 36 to stand for the color number of the three vertexes related with two immovable edges; the other kind of graphs is the structure with only one 3-cycle formed by two immovable edges and the tree like structure, also the color functions of these structures are all added to get one with coefficient $b$ and 24 for the color number of the 3-cycle as shown in figure 4 (a) and (b) below.

$<$ Figure $4>$

From figure 4(a), we get the color function below:

$$
P_{4}=[36 a]_{A}+\left[(-1)^{n} 24 b\right]_{B}=36 a-24 b
$$

(here $a=3, b=1$ and $n=3$ ).

From figure 4(b), we get the color function below:

$$
P_{5}=[36 a]_{A}+\left[(-1)^{n} 24 b\right]_{B}=36 a+24 b
$$

(here $a=6, b=1$ and $n=2$ ).

Here we divide the function into two parts. One with the number 36 is regarded as part A; and the other with the number 24 is regarded as part $B$.

As shown in figure 5, just add one edge on the left and right sides of the two immovable edges in figure 4(a). In this case one kind of graphs belong to the part A has one edge to be added to form a 3-cycle; the other kind of graphs for part B has no changes, because the overlap edge is added. So we get the function below:

$$
P_{+e}=24 a-24 b
$$

(here $\mathrm{a}=3, \mathrm{~b}=1$ )

$<$ Figure $5>$

\subsection{Example of discompose for 4-cycle structure with the method of immovable edges}

Figure 6 shows the graph $T$ with one vertex which is removed. This removed vertex is located inside a 4-cycle (In order to be convenient the vertices and edges outside are not shown).

$<$ Figure $6>$

Using the same method introduced above, we get the color function which is shown below:

$$
P(T-v)=P=[36 a]_{A}+\left[(-1)^{n} 24 b\right]_{B}>0
$$

(here $a, b$ and $n$ are whole numbers)

Here the vertexes of $P$ are less than graph $T$, so the color number for graph $T$ is bigger than zero. Then we add one edge inside the 4-cycle, as shown in figure 7 below.

$<$ Figure $7>$

We get the color function which is shown below:

$$
P_{+e}=[24 a]_{A}+\left[(-1)^{n} 24 b\right]_{B}>0
$$

Here $P_{+e}$ is also bigger than zero. When $n$ is odd number, we get $\mathrm{a}>\mathrm{b}$.

Change the place of immovable edges as shown in figure 8 below:

$<$ Figure $8>$ 
We get the color function which is shown below:

$$
P=\left[36 k_{1} a\right]_{A}+\left[(-1)^{n} 24 k_{2} b\right]_{B}>0
$$

Then we add one edge inside the 4-cycle, as shown in figure 9 below.

$<$ Figure $9>$

We get the color function which is shown below:

$$
P_{+e}=\left[24 k_{1} a\right]_{A}+\left[(-1)^{n} 24 k_{2} b\right]_{B}>0
$$

Equation (2.6)-(2.7), we get:

$$
\begin{gathered}
P_{-v}=P-P_{+e} \\
=\left[36 k_{1} a\right]_{A}+\left[(-1)^{n} 24 k_{2} b\right]_{B}-\left[24 k_{1} a\right]_{A}-\left[(-1)^{n} 24 k_{2} b\right]_{B}=12 k_{1} a>0
\end{gathered}
$$

The result can be shown by the graphs below in figure 10 .

$<$ Figure $10>$

We add one vertex and two edges inside the 4-cycle, as shown in figure 11 below.

$<$ Figure $11>$

In this case, the vertex inside the 4-cycle has two kinds of colors to be colored, so the color function is as below:

$$
P_{+v+2 e}=[72 a]_{A}+\left[(-1)^{n} 48 b\right]_{B}
$$

Then we add one edge more inside the 4-cycle, as shown in figure 12 below.

$<$ Figure $12>$

Here we get

$$
\begin{gathered}
P_{1}=[72 a]_{A}+\left[(-1)^{n} 48 b\right]_{B} \\
P_{2}=\left[24 a+(-1)^{n} 24 b\right]_{B}
\end{gathered}
$$

Hence we get

$$
\begin{gathered}
P_{+v+3 e}=P_{1}-P_{2}=[72 a]_{A}+\left[(-1)^{n} 48 b\right]_{B}-\left[24 a+(-1)^{n} 24 b\right]_{B} \\
=[72 a]_{A}+\left[(-1)^{n} 24 b-24 a\right]_{B}
\end{gathered}
$$

Still then we add one edge more inside the 4-cycle, as shown in figure 13 below.

$<$ Figure $13>$

Here we get

$$
\begin{gathered}
P_{1}=[72 a]_{A}+\left[(-1)^{n} 24 b-24 a\right]_{B} \\
P_{2}=[36 a]_{A}+\left[(-1)^{n} 24 b\right]_{B} \\
P_{3}=12 k_{1} a \\
P_{+v+4 e}=P_{1}-P_{2}+P_{3} \\
=[72 a]_{A}+\left[(-1)^{n} 24 b-24 a\right]_{B}-[36 a]_{A}-\left[(-1)^{n} 24 b\right]_{B}+12 k_{1} a \\
=[36 a]_{A}-[24 a]_{B}+12 k_{1} a=12 a+12 k_{1} a>0
\end{gathered}
$$

\section{Conclusion}

The configuration of 4-cycle in $T$ graph is reducible or it does not exist in the $T$ graph. This is an another method compare with the one which has already carried out by others.

\section{Reference}

Douglas B. West. (2006). Introduction to Graph Theory, pp. 206.

Gray Chartrand, Ping Zhang. (2007). Introduction to Graph Theory, pp. 234-236.

Wang S-H. Graph Theory. (2004). Beijing Science Publishing House, pp. 96-97.

Yu Qiu. (2010, 7). Invalid Counterexample in Graph Theory. [Online] Available: http://www.paper.edu.cn Yu Qiu. (2010). Invalid Counterexample in Graph Theory. Journal of Mathematics Research, 2(4), pp. $130-134$. 


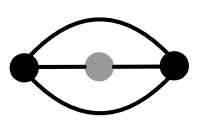

(a)

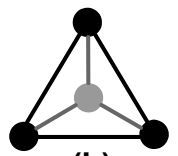

(b)

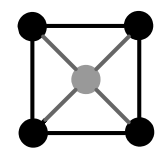

(c)

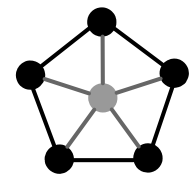

(d)

Figure 1. Unavoidable complete set

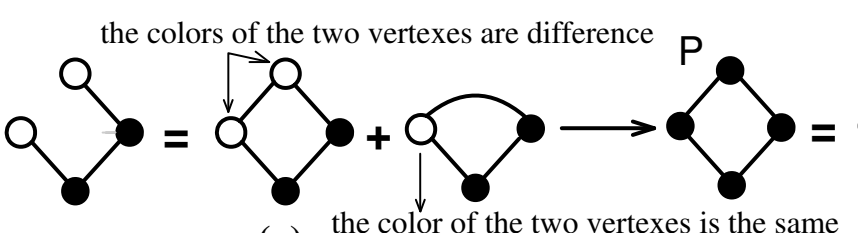

(a) the color of the two vertexes is the same

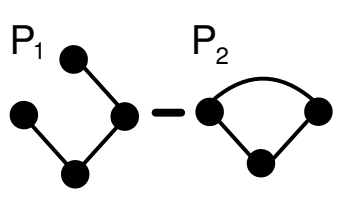

(b)

Figure 2. Discompose methods of similarity and difference

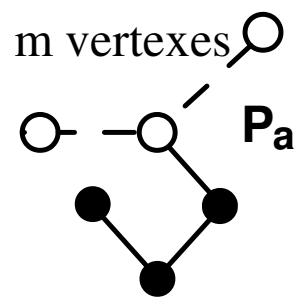

(a)

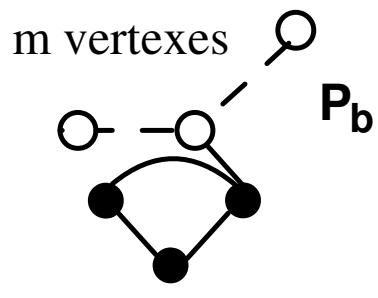

(b)

Figure 3. Graphs with the structure of tree alike

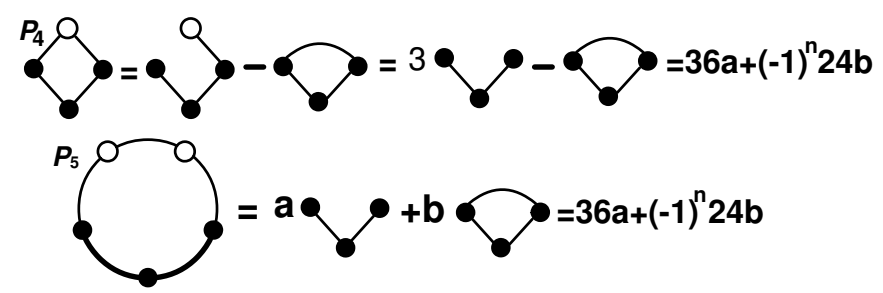

Figure 4. Discompose examples of 4-cycle and 5-cycle

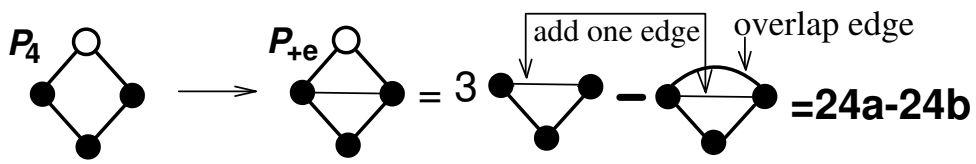

Figure 5. Adding one edge on the left and right sides of the two immovable edges

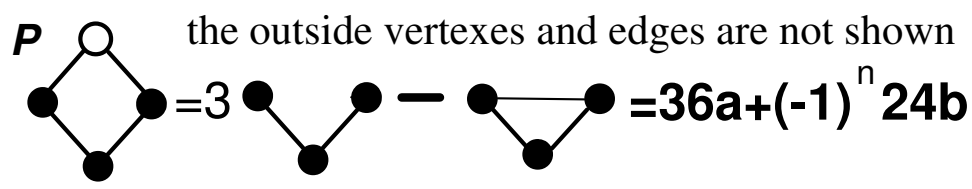

Figure 6. Discompose of $T-v$ graph

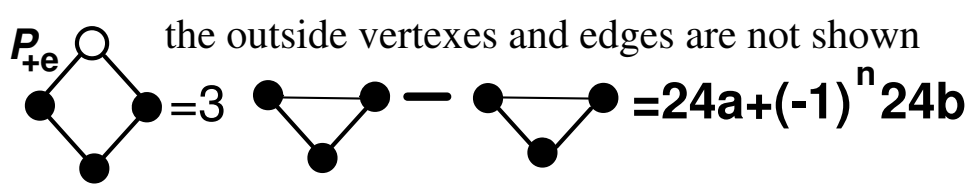

Figure 7. Discompose of $T-v$ graph 


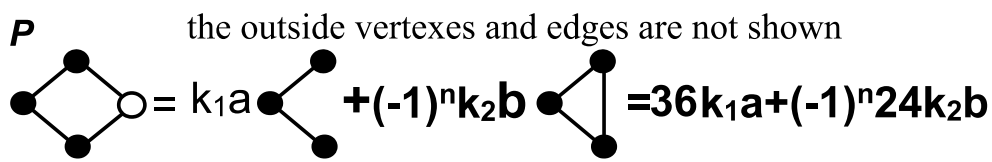

Figure 8. Discompose of $T$ - $v$ graph

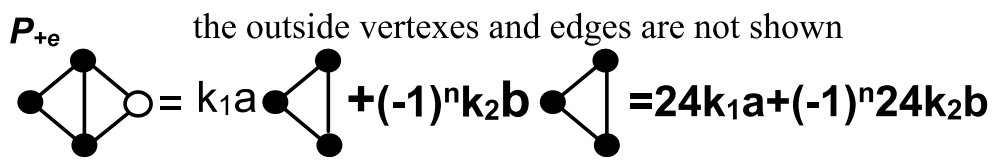

Figure 9. Discompose of $T-v$ graph

the outside vertexes and edges are not shown

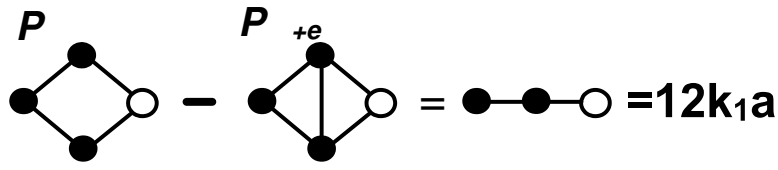

Figure 10. Result graphs to be shown

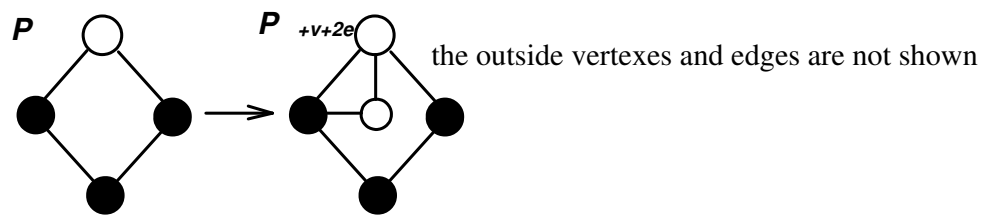

Figure 11. Adding one vertex and two edges inside the 4-cycle

the outside vertexes and edges are not shown

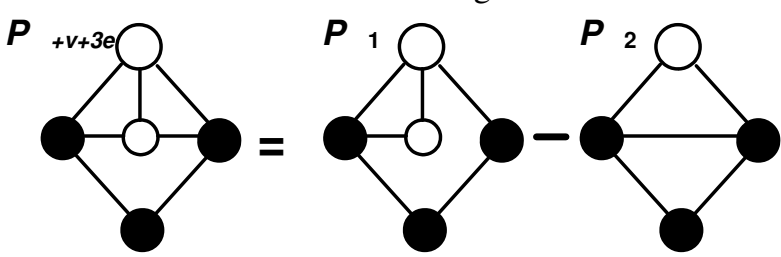

Figure 12. Adding one vertex and three edges inside the 4-cycle

the outside vertexes and edges are not shown
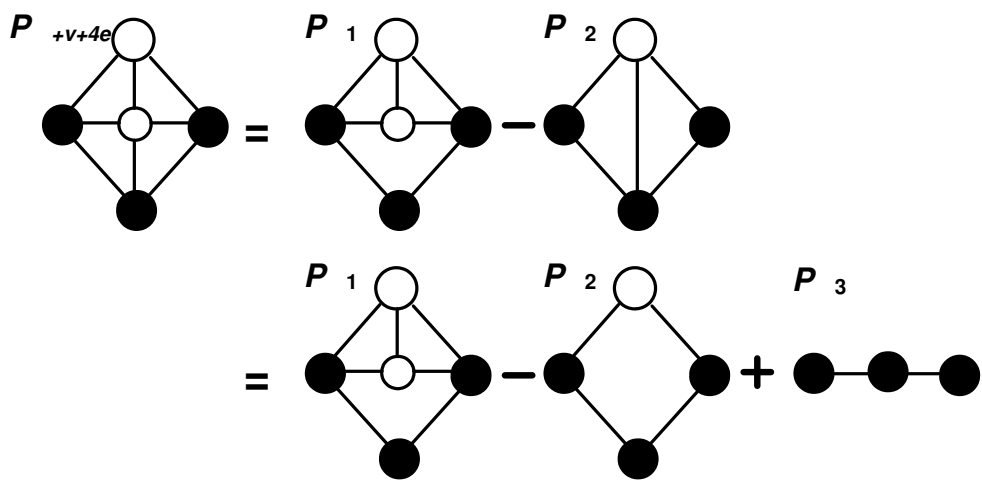

Figure 13. Adding one vertex and four edges inside the 4-cycle 